that the next 50 years will bring a genuine revolution of far greater individual significance than that delivered by genetics over the past 50 years. This is because lifestyle can conceivably be analysed, and in so doing, it should be possible to develop a genuinely personalized medicine.

Researchers can now think seriously about how to identify lifestyle influences: such studies will have to be on an unprecedented scale and one of the first of these, proposed to comprise 500,000 individuals in the United Kingdom, has already started ${ }^{16}$. These kinds of studies are a bold venture into relatively uncharted territory and face substantial technical, biological and scienceculture challenges.

Scientifically, it is necessary to understand a deceptively simple equation: genes + environment $=$ outcome. The difficulty here is the uncertainty surrounding both terms in the equation; ideally, one set of genetic factors will interact with one set of environmental influences to produce identical outcomes, but it is unknown whether this is always going to be the case. A far more difficult relationship would exist if multiple genetic factors interacted with multiple environments to achieve the same outcome. The example of glutathione $S$-transferase mutations, smoking and incidence of lung cancer ${ }^{17}$ shows it is possible to detect some interactions, but it is unclear how, or even if, statistical methods might be developed for addressing the more complex possibilities.

Perhaps the greatest unknown in undertaking these projects is human psychology; the consequences of smoking have been known for many decades, but people still smoke. Advice does not imply acceptance. How to turn knowledge into practical outcomes must be an increasing focus of attention for both researchers and funding agencies.

Psychology is also in play in the initial decision to undertake this research; for researchers, funding agencies and politicians there is great risk implicit in undertaking a hugely expensive project with complex outcome. People would like to live in a simpler world, with simpler decisions, but the vision of such a project is enormous: once complete, as much will be known about the origins of human disorders as can be discovered by using such epidemiological and genetic studies. Perhaps more important, the beginnings of a new medicine will emerge, one focused uniquely and completely upon the individual, upon the combination of genetic uniqueness and personal choices that are the very essence of individual lives.

If we are collectively bold in our present decisions and accept the risk of action, a world can be created where medicine is a guide, not a place of last resort. If the past 50 years has seen the revolution of DNA, then the revolution cannot be completed without an appreciation of both genetic and environmental individuality; only then will individuals understand the meaning of their inheritance.

\section{doi:10.1038/nature 01401}

1. Jaroff, L. The gene hunt. Time 20 March, 62-67 (1989)

2. Arcos-Burgos, M. \& Muenke, M. Genetics of population isolates. Clin. Genet. 61, 233-247 (2002)

3. Aidoo, M. et al. Protective effects of the sickle cell gene against malaria morbidity and mortality. Lancet 359, 1311-1312 (2002).

4. Tishkoff, S. A. \& Williams, S. M. Genetic analysis of African populations: human evolution and complex disease. Nature Rev. Genet. 3, 611-621 (2002).

5. Chakravarti, A. Single nucleotide polymorphisms: ...to a future of genetic medicine. Nature 409, 822-823 (2001).

6. Carrasquillo, M. M. et al. Genome-wide association study and mouse model identify interaction between RET and EDNRB pathways in Hirschsprung disease. Nature Genet. 32, 237-244 (2002).

7. Cox, N. J. Challenges in identifying genetic variation affecting susceptibility to type 2 diabetes: examples from studies of the calpain-10 gene. Hum. Mol. Genet. 10, 2301-2305 (2001).
8. Sullivan, P. F. et al. Analysis of epistasis in linked regions in the Irish study of high-density schizophrenia families. Am. J. Med. Genet. 105, 266-270 (2001).

9. Boyle, J. P. et al. Projection of diabetes burden through 2050: impact of changing demography and disease prevalence in the United States. Diabetes Care 24, 1936-1940 (2001).

10. Münger, K. Disruption of oncogene/tumor suppressor networks during human carcinogenesis. Cancer Invest. 20, 71-81 (2002).

11. Panda, S., Hogenesch, J. B. \& Kay, S. A. Circadian rhythms from flies to human. Nature 417, 329-335 (2002).

12. MacGregor, A. J. et al. Twins: novel uses to study complex traits and genetic diseases. Trends Genet. 16, 131-134 (2000).

13. Wertz, D. C. Ethics watch. Nature Rev. Genet. 3, 496 (2002).

14. Evans, W. E. \& Johnson, J. A. Pharmacogenomics: the inherited basis for interindividual differences in drug response. Annu. Rev. Genomics Hum. Genet. 2, 9-39 (2001).

15. Lown, K. S. et al. Grapefruit juice increases felodipine oral availability in humans by decreasing intestinal CYP3A protein expression. J. Clin. Invest. 99, 2545-2553 (1997).

16. Wright, A. F., Carothers, A. D. \& Campbell, H. Geneenvironment interactions- the BioBank UK study. Pharmacogenomics J. 2, 75-82 (2002).

17. Stucker, I. et al. Genetic polymorphisms of glutathione Stransferases as modulators of lung cancer susceptibility. Carcinogenesis 23, 1475-1481 (2002).

\title{
The double helix in clinical practice
}

\section{John I. Bell}

The Office of the Regius Professor of Medicine, University of Oxford, Oxford OX3 9DU, UK (e-mail:regius@medsci.ox.ac.uk)

The discovery of the double helix half a century ago has so far been slow to affect medical practice, but significant transformations are likely over the next $\mathbf{5 0}$ years. Changes to the way medicine is practised and new doctors are trained will be required before potential benefits are realized.

"It is much more important to know what kind of patient has a disease than to know what kind of disease a patient has." Caleb Parry, 18th century physician, Bath.

$\mathrm{T}$ he structure of DNA established the basic framework that would develop into the field of molecular genetics. The information gleaned from this scientific endeavour continues to have a profound influence on our understanding of biological systems ${ }^{1}$. As most human diseases have a significant heritable component, it was soon recognized that the characterization of the genetic determinants of disease would provide remarkable opportunities for clinical medicine, potentially altering the way disease was understood, diagnosed and treated.

But despite the obvious potential applications to medicine, the development of significant genetic advances relevant to clinical practice could take generations. This is in marked contrast to many other medically related discoveries that occurred around the same time and which were translated rapidly into clinical practice. For instance, the development of penicillin by Ernst Chain and Howard Florey in 1941 was saving thousands of lives within months of their discovery of how to efficiently produce the antibiotic $^{2}$. Discoveries relating to disease aetiology, such as the recognition in 1950 of a relationship between smoking and lung cancer, have had a profound effect on mortality ${ }^{3}$.
This was despite the convictions of at least one distinguished statistical geneticist who argued against the causality of this observation, implying that a common genetic factor caused both lung cancer and a predilection to smoking cigarettes ${ }^{4}$ !

Although other important discoveries have had demonstrably more impact on health care at the time of their fiftieth anniversaries than has the double helix, its slower transition from discovery to clinical implementation will be balanced by its potentially profound impact across all medical disciplines. Progress has been slow, but mounting evidence suggests that, while public health and antibiotics produced important healthcare outcomes in the past 50 years, the next 50 are likely to belong to genetics and molecular medicine.

The potential impact of genetics on clinical practice has been questioned by some observers $^{5}$ who believe that the positive predictive value of genetic testing for most common disease genes will be insufficient to provide the beneficial effects seen with single-gene disorders, which affect only a tiny proportion of the population. Many advocates of genetics argue, on the other hand, that our understanding of disease is 


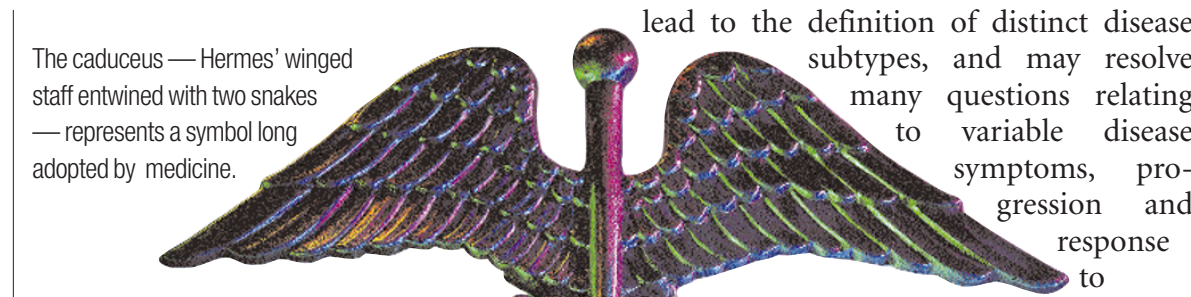

undergoing a major change. They contend that genetic research is playing a fundamental role in improving our understanding of the pathophysiology that underlies disease and that, inevitably, as this is applied, it will alter both the theory and practice of medicine in the future ${ }^{6}$.

A new taxonomy for human disease Clinical practice has always been limited by its inability to differentiate clinical, biochemical and pathological abnormalities that accompany a disease from those events actually responsible for mediating a disease process. Clinicians may have moved on from calling 'fever' a disease ${ }^{7}$, but they still rely on phenotypic criteria to define most diseases, and yet these may obscure the underlying mechanisms and often mask significant heterogeneity. As Thomas Lewis pointed out in 1944, diagnosis of most human disease provides only "insecure and temporary con-

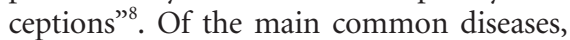
only the infectious diseases have a truly mechanism-based nomenclature.

An understanding of the genetic basis of maladies is providing a new taxonomy of disease, free from the risk that the diagnostic criteria related to events are secondary to the disease process, rather than to its cause. Genetic information has allowed us to identify mechanistically distinct forms of diabetes, defining an autoimmune form of the disease associated with human leukocyte antigens (a highly diverse complex of immune-system genes), and recently has implicated dysfunction of factors that affect both expression and modification of gene products in mediating the adult form of the disorder ${ }^{9}$. Similarly, we are now aware of a range of molecules and pathways previously not recognized in the pathogenesis of asthma $^{10-12}$.

A clearer understanding of the mechanisms and pathways that mediate disease will

\begin{tabular}{l}
\hline Table 1 Molecular genetics in clinical practice \\
- Mechanistically based diagnostic criteria \\
- Predisposition testing and screening \\
- Rapid molecular diagnostic testing of pathogens \\
- Pharmacogenetics \\
- Identification of new drug targets \\
- Tools for molecular medicine (for example, recombinant \\
DNA methodology) \\
- Recombinant expression of therapeutic proteins \\
- Gene therapy
\end{tabular}

Molecular biology has improved our ability to study proteins and pathways involved in disease and has provided the technology necessary to generate new sets of targets for smallmolecule drug design. It has also enabled the creation and production of a new range of biological therapeutics - recombinant proteins such as interferon, erythropoietin and insulin, as well as therapeutic antibodies, which are one of the fastest growing classes of new treatments. Further extensions of this methodology will see the inevitable introduction of DNA-based therapies that will produce proteins of interest in the appropriate cellular setting. DNA-based vaccines represent the first wave of such novel gene-therapy approaches to disease and many more are expected to follow.

We are undergoing a revolution in clinical practice that depends upon a better understanding of disease mechanisms and pathways at a molecular level. Much has already been achieved: an enhanced understanding of disease-related pathways, new therapies, novel approaches to diagnostics and new tools for identifying those at risk. But more remains to be done before the full impact of genetics on medicine is realized. Complex disease, with multiple susceptibility determinants (both environmental and genetic), will take time to dissect. This information must then be moved into the clinic and evaluated for its benefits.

As the practice of medicine moves to one more scientifically founded in disease mechanisms, many aspects of clinical practice will need to be transformed. Individual genetic variation is likely to explain a significant part of the heterogeneity seen clinically in the natural history of disease and in response to therapy. Tools to tailor medicine to an individual's needs rather than directing it at a population will inevitably become available. Similarly, as predictions of risk improve, early or preventative therapy of high-risk populations will become a reality, with screening programmes targeted to those at particularly high risk.

\section{Transforming clinical practice}

For fundamental changes to take place in clinical practice, sweeping transformation will be needed to healthcare provision, economic management and training. It is currently difficult to predict the cost-benefit ratio for such changes - certainly the present impact of molecular medicine has not made medicine less expensive. Few medical schools adequately train their students to think mechanistically about disease; indeed, the trend towards pattern-recognition medicine, away from basic science training, means that we are still far from educating the next generation of clinicians to apply the knowledge and tools bequeathed to us by the double helix. The evolution in health care that will incorporate these new principles of early diagnosis and individualized therapy will be a daunting 
challenge in an era of uncertainty for healthcare systems worldwide.

The influence of genetic and molecular medicine on the health of patients is already sufficiently ubiquitous that it will have an impact on most common diseases. Its influence will grow over the next few decades (Table 1). It will not, however, answer all of the questions about human health, nor will it provide all the answers for optimizing clinical practice. The reductionism that accompanies molecular genetics will identify the pieces in the jigsaw, but assembling these to understand how complex systems malfunction will require a substantially more integrated approach than is available at present.

The crucial role played by environmental determinants of disease will perhaps become more tractable when combined with an understanding of genetic susceptibility. Sceptics, rightly, will wish to see more data before they acknowledge that molecular medicine will be truly transformed over the next 50 years, despite the fact that its influence on diagnostics and new therapeutics is already clearly apparent. A transition is underway, the direction of travel is clear, but managing the change in clinical practice may prove at least as challenging as resolving the original structure of the helix.

doi:10.1038/nature01402

1. Watson, J. D. \& Crick, F. C. H. A structure for deoxyribose nucleic acid. Nature 171, 737-738 (1953).

2. Abraham, E. P. et al. Further observations on penicillin. Lancet ii, 177-188 (1941)

3. Doll, R. \& Hill, A. B. Smoking and carcinoma of the lung. Br. Med. J. 2, 739-748 (1950).

4. Fisher, R. A. Cancer and smoking. Nature 182, 596 (1958).

5. Holtzman, N. A. \& Marteau, T. M. Will genetics revolutionise medicine? N. Engl. J. Med. 343, 141-144 (2000).

6. Bell, J. I. The new genetics in clinical practice. Br. Med. J. $\mathbf{3 1 6}$ 618-620 (1998).

. Osler, W. The Principles and Practice of Medicine (Appleton, $\mathrm{New}$ York, 1892).

8. Lewis, T. Reflections upon medical education. Lancet i, 619-621 (1944).

9. Cardon, L. R. \& Bell, J. I. Association study designs for complex diseases. Nature Rev. Genet. 2, 91-99 (2001).

10. Van Eerdewegh, P. et al. Association of the ADAM33 gene with asthma and bronchial hyperresponsiveness. Nature 418, 426-430 (2002).

11. Cookson, W. O. C. M., Sharp, P. A., Faux, J. A. \& Hopkin, J. M. Linkage between immunoglobulin $\mathrm{E}$ responses underlying asthma and rhinitis and chromosome 11q. Lancet 1, 1292-1295 (1989).

12. Shirakawa, I. et al. Atopy and asthma: genetic variants of IL-4 and IL-13 signalling. Immunol. Today 21, 61-64 (2000).

13. Pharoah, P. D. P. et al. Polygenic susceptibility to breast cancer and implications for prevention. Nature Genet. 31,33-36 (2002).

14. Splawski, I. et al. Variant of SCN5A sodium channel implicated in risk of cardiac arrhythmia. Science 297, 1333-1336 (2002).

15. Weber, W. W. Pharmacogenetics (Oxford Univ. Press, New York, 1997)

\section{The Mona Lisa of modern science}

\section{Martin Kemp}

Department of the History of Art, University of Oxford, Littlegate House, St Ebbes, Oxford OX1 1PT, and Wallace Kemp, Artakt, Studio D, 413-419, Harrow Road, London W93QJ, UK (e-mail: martin.kemp@trinity.ox.ac.uk)

No molecule in the history of science has reached the iconic status of the double helix of DNA. Its image has been imprinted on all aspects of society, from science, art, music, cinema, architecture and advertising. This review of the Mona Lisa of science examines the evolution of its form at the hands of both science and art.

"A monkey is a machine that preserves genes up trees, a fish is a machine that preserves genes in water; there is even a small worm that preserves genes in German beer mats. DNA works in mysterious ways." Richard Dawkins in The Selfish Gene (Oxford University Press, 1976).

$\mathrm{H}$ istory has thrown up a few superimages, which have so insinuated themselves into our visual consciousness that they have utterly transcended their original context. This is epitomized by the Mona Lisa, painted by Leonardo da Vinci around 1503. The double helix of DNA is unchallenged as the image epitomizing the biological sciences. Both images speak to audiences far beyond their respective specialist worlds, and both carry a vast baggage of associations.

In the worlds of popular image diffusion, particularly on the Internet, the double helix is beginning to rival the Mona Lisa as a playground for eccentrics and obsessives (Fig. 1). There is an apparent difference, of course. Leonardo's panel painting is the product of human artifice, whereas DNA is a naturally occurring, large organic molecule. But Leonardo claimed that his art represented a systematic remaking of nature on the basis of a rational understanding of causes and effects. His painting is the result of a complex, nonlinear interaction between concept, subject, plan of action, acquired knowledge, skill, medium and the evolving image itself. In The Art of Genes ${ }^{1}$, Enrico Coen argues that "biological development and human creativity are highly interactive processes in which events unfold rather than being necessarily pre-planned or anticipated. In other words, in both cases there is no easy separation between plan (or programme) and execution."

Looking at the investigation and representations of the double helix, we can say that

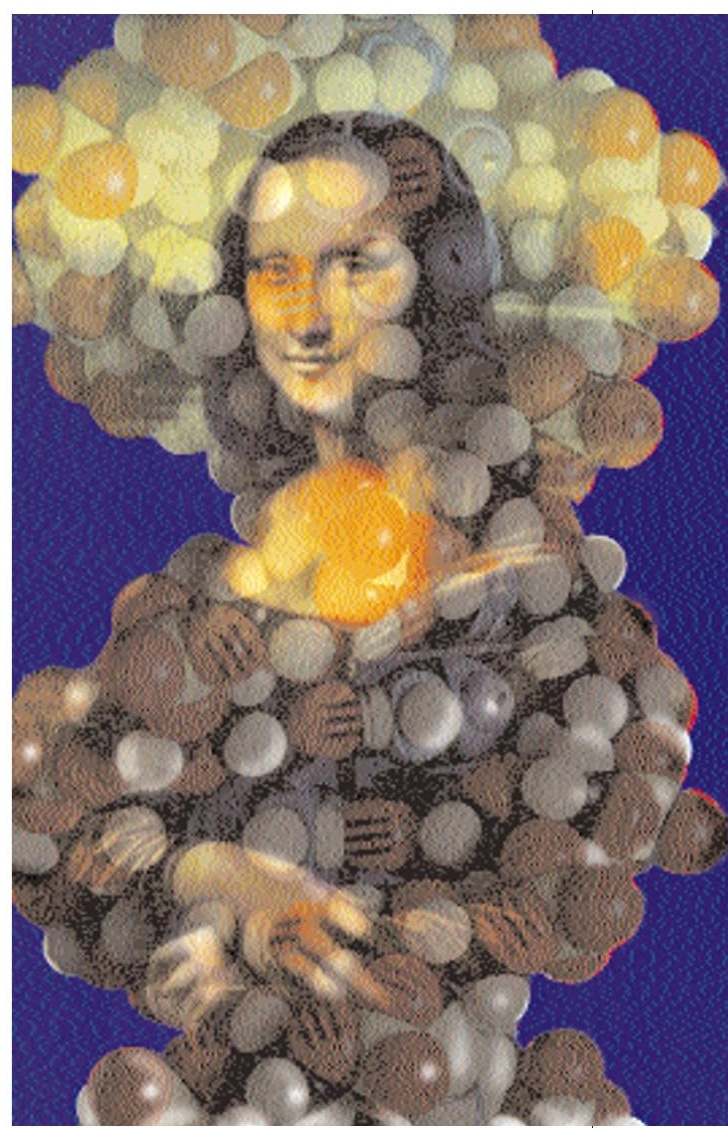

they are cultural activities no less than any painting. Behind the discovery lies the vast infrastructure of a scientific culture that led to the development of the knowledge, theories, institutions, techniques and equipment that made the quest both possible and desirable. The very natures of scientific models and representations, using whatever technique, are integral to the vehicles of science communication. Their visual look is compounded from a complex set of factors, ranging from technical to aesthetic. But, in case anyone should be getting the wrong impression, I acknowledge that the cultural vehicles are designed to deliver nonarbitrary information that is open to rational scrutiny as a way of working towards real knowledge of the physical constitution of the world.

Looked at from a popular perspective (and even from the standpoint of reputation within science), James Watson and Francis Crick are identified with DNA no less than Leonardo is identified with the Mona Lisa. The researchers were in a very real sense the 'authors' or 'artists' of the acts of visualization that generated their models of the molecule. But their brilliant achievement was not necessarily of a higher order than that of the other pioneers of molecular modelling, such as the Braggs, John Kendrew, Max Perutz, Maurice Wilkins and Linus Pauling. Rather, they were uniquely fortunate that their molecule was both visually compelling, as a supreme example of nature's 'sculpture', and 